\title{
Indocyanine green angiography in inflammatory eye disease
}

\begin{abstract}
Purpose The choroid plays an integral role in the evolution of a number of inflammatory eye diseases but only limited information is provided on the choroidal vasculature by fluorescein angiography.

Methods This study used indocyanine green (ICG) videoangiography to assess the degree of choroidal vascular involvement in inflammatory eye diseases and the results obtained were compared with fluorescein angiography.

Results A total of 34 patients were examined: 6 with retinal vasculitis and 28 with chorioretinitis of various aetiologies. Areas of active involvement of the choroid were seen as areas of hypofluorescence on ICG angiography, representing areas of inflammatory infiltrate and/or choroidal hypoperfusion. No leakage of ICG occurred from large choroidal vessels at any stage in any disease. In contrast to sodium fluorescein, there was no leakage of ICG from retinal vessels in active inflammation. An inflammatory choroidal neovascular membrane leaked fluorescein and fluoresced late with ICG.

Conclusion ICG videoangiography provides useful information on the degree of choroidal involvement in inflammatory eye disease and is a valuable adjunct to fluorescein angiography.
\end{abstract}

Key words Choroid, Fluorescein angiography, Indocyanine green angiography, Uveitis

Inflammatory eye disease continues to be a significant cause of severe visual loss, particularly amongst young adults. ${ }^{1,2}$ This is despite increased understanding of the complex mechanisms that underlie the pathogenesis of uveitis. ${ }^{3-5}$ The choroidal vasculature is known to be intimately involved in the inflammatory process $^{6-9}$ but is difficult to study in vivo as only limited information is provided by fluorescein angiography. High-quality images of the choroidal vessels can now be obtained using indocyanine green (ICG) as the contrast
LUCY HOWE, MILES STANFORD,

ELIZABETH GRAHAM, JOHN MARSHALL medium in conjunction with a specially adapted fundus camera ${ }^{10}$ or scanning laser ophthalmoscope. ${ }^{11}$

This study was carried out to assess the extent and type of choroidal involvement in a variety of inflammatory eye diseases in man using ICG choroidal angiography.

\section{Methods}

This study was approved by the hospital ethics committee and informed written consent was obtained from all patients for inclusion in the study. Patients with posterior uveitis were recruited from the specialist uveitis clinics and Medical Eye Unit of St Thomas' Hospital, London. Patients with retinal vasculitis had symptoms of visual disturbance with vitreous cells (with or without anterior chamber activity) and retinal inflammatory signs of periphlebitis, macular oedema and/or swelling of the optic disc. Patients with active choroiditis had symptoms of visual disturbance with vitreous cells (with or without anterior chamber activity) and lesions evident ophthalmoscopically that appeared to be at the level of the choroid. Patients were diagnosed as having sarcoidosis on the basis of a compatible clinical history and examination combined with a positive tissue biopsy and/or serum angiotensin converting enzyme level of $\geqslant 90$ IU (St Thomas' Hospital normal reference range 16-53 IU). Patients were excluded if they were pregnant or had a known allergy to iodine, as the commercial preparation of ICG used in this study, Cardio Green (Paesel and Lorei, Frankfurt, Germany), contains iodine.

Ophthalmic examination was followed by colour fundal photography and angiography. The majority of patients had fluorescein angiography performed at the same time as ICG angiography, while a few had ICG angiography alone. Fluorescein angiography was performed with a standard fundus camera (Kowa Pro-1, Tokyo, Japan) or with a scanning laser ophthalmoscope (model 101, Rodenstock, Munich, Germany) using the argon laser. ICG angiography was performed with $50 \mathrm{mg}$ of ICG on both eyes of all patients using the infrared

\author{
L. Howe \\ J. Marshall \\ Department of \\ Ophthalmology \\ UMDS \\ London, UK \\ M. Stanford \\ E. Graham \\ Medical Eye Unit \\ St Thomas' Hospital \\ London, UK \\ L. Howe, FRCOphth \\ The Western Eye Hospital \\ Marylebone Road \\ London NW1 5YE, UK \\ Tel: +44 (0)171 8866666 \\ Fax: +44 (0)171 8863270
}




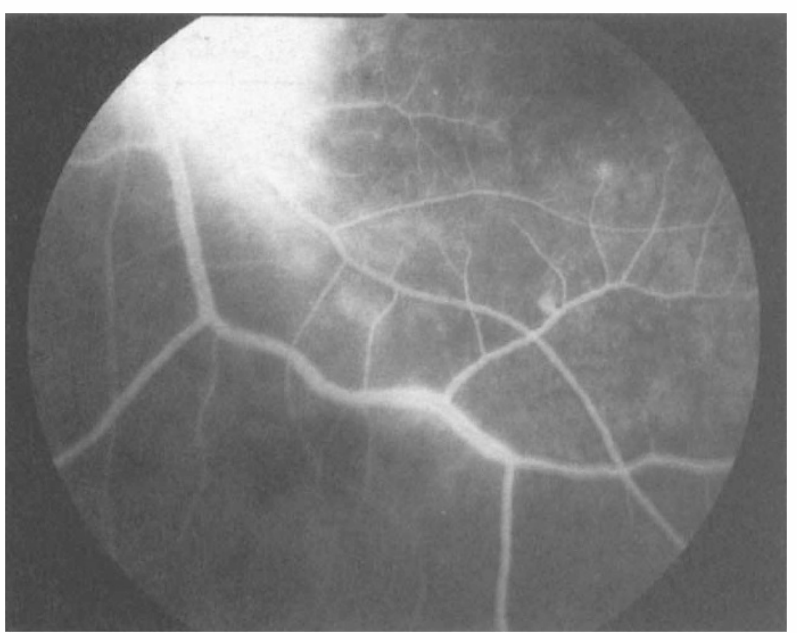

Fig. 1. Case 1: idiopathic chorioretinitis. Late fluorescein angiogram of the left inferotemporal arcade and optic disc showing marked fluorescein leakage from the disc and retinal vein.

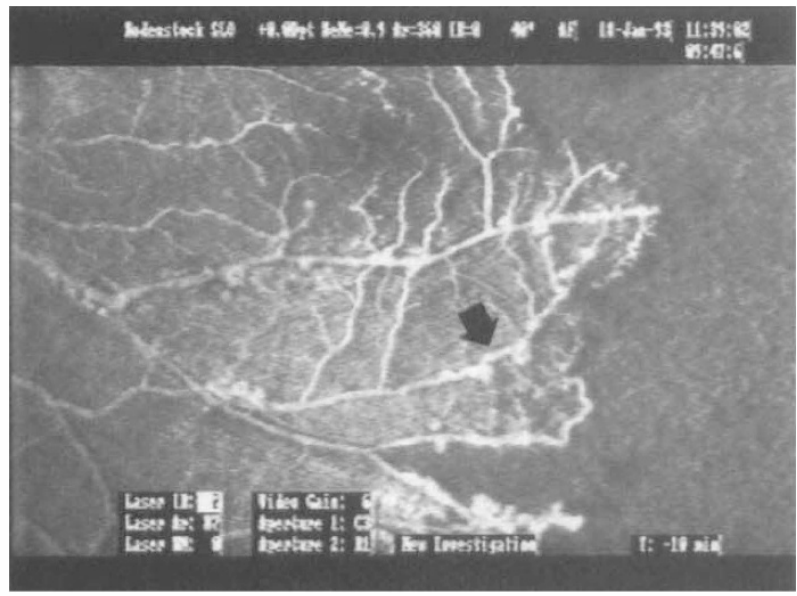

Fig. 3. Case 2: severe ischaemic retinal vasculitis. Fluorescein angiogram of the area temporal to the left macula showing marked retinal capillary drop-out in the periphery.

diode laser of the scanning laser ophthalmoscope. Pictures were taken for the first $5 \mathrm{~min}$ of the ICG angiogram, then at $10 \mathrm{~min}$ and at $30-40 \mathrm{~min}$. Data were recorded on U-matic videotape and specific images were captured using a framegrabber.

\section{Results}

A total of 34 patients were recruited into the study: 14 men and 20 women with a mean age of 44 years (range 21-75 years). The study group consisted of 27 Caucasians, 3 Afro-Caribbeans and 4 Asians. The diagnoses included 6 patients with retinal vasculitis and 28 with chorioretinitis of various aetiologies (Table 1). Table 2 gives brief details of the patients chosen to illustrate various angiographic findings (Figs. 1-12).

Table 3 summarises the angiographic findings in all the patients studied with retinal signs and includes the findings in the subgroup of 6 patients with a diagnosis of retinal vasculitis. Fluorescein leakage was detected in areas of periphlebitis, macular oedema, from vessels

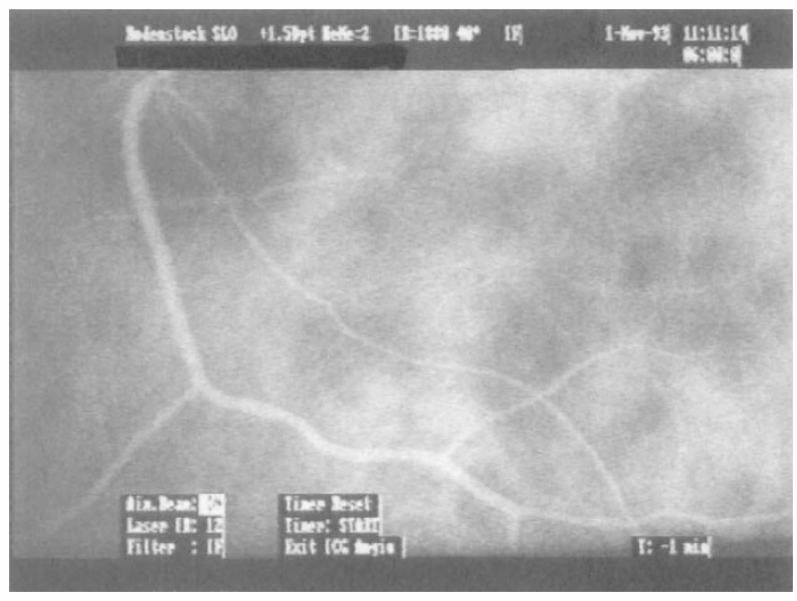

Fig. 2. Case 1: idiopathic chorioretinitis. ICG angiogram of the same area as Fig. 1 showing no dye leakage.

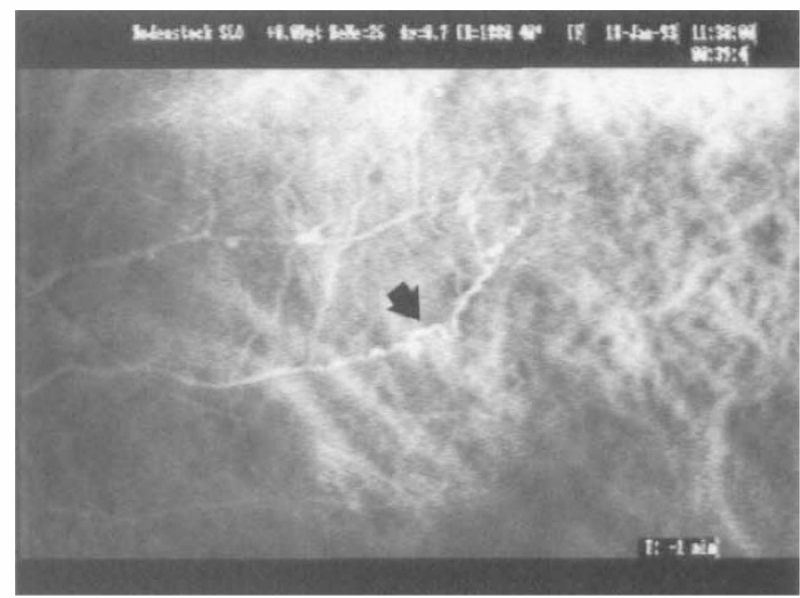

Fig. 4. Case 2: severe ischaemic retinal vasculitis. ICG angiogram of the same area as in Fig. 3 showing normal underlying choroidal vessel filling. Arrow indicates same retinal vessel as in Fig. 3.

traversing areas of retinal ischaemia and from swollen optic discs. Structural abnormalities of the retinal arteries and veins were demonstrated by ICG angiography but there was no leakage of ICG from any retinal vessels (compare Figs. 1 and 2, case 1). One patient had ICG staining of a segment of inferonasal retinal vein but the significance of this is uncertain. The choroidal vessels of all the patients with a diagnosis of retinal vasculitis appeared normal and the choroidal vasculature underlying areas of retinal ischaemia appeared intact (case 2, Figs. 3 and 4).

Table 1. Summary of patient diagnoses

\begin{tabular}{lcl}
\hline Diagnosis & No. of patients & Further details \\
\hline Retinal vasculitis & 6 & 2 sarcoidosis, \\
& & $\begin{array}{l}\text { 2 ischaemic, } \\
\text { 2 idiopathic }\end{array}$ \\
Idiopathic chorioretinitis & 18 & \\
Sarcoid choroiditis & 3 & \\
Multifocal choroiditis & 5 & \\
Toxoplasma chorioretinitis & 1 & \\
Sympathetic ophthalmitis & 1 & \\
\hline
\end{tabular}




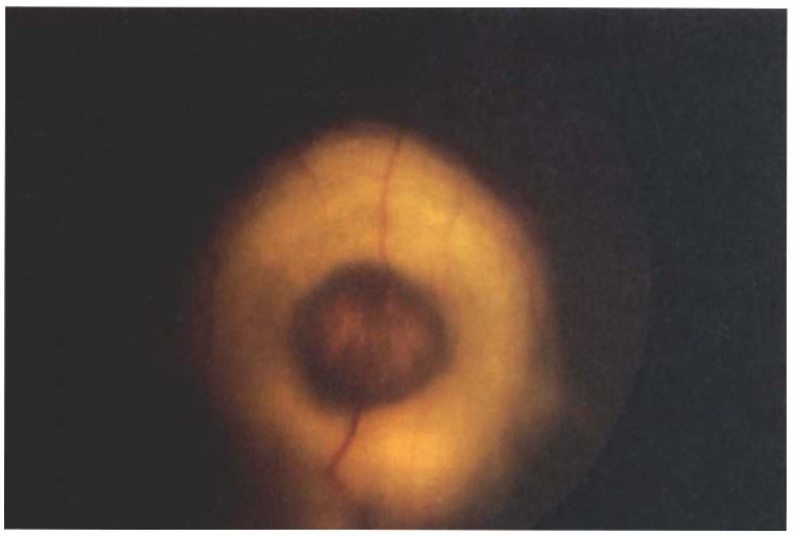

Fig. 5. Case 3: idiopathic choroiditis. Colour photograph of right fundus showing elevated doughnut-shaped choroidal lesion.

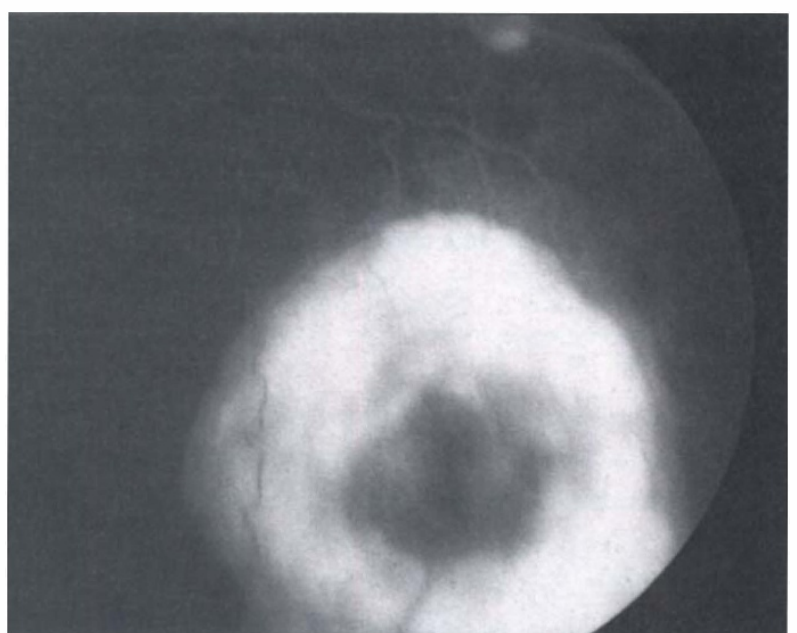

Fig. 6. Case 3: idiopathic choroiditis. Fluorescein angiogram in the late venous phase showing hyperfluorescence of the doughnut-shaped choroidal lesion shown in Fig. 5.

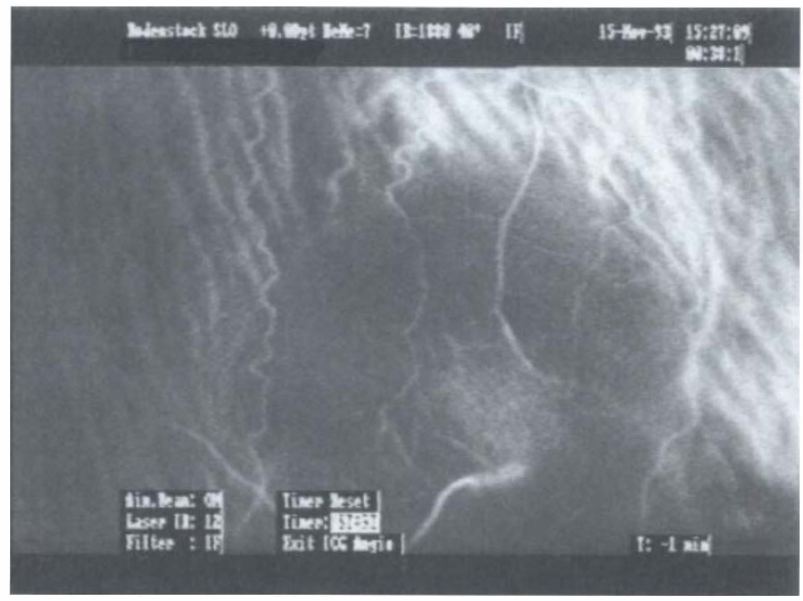

Fig. 7. Case 3: idiopathic choroiditis. ICG angiogram of same lesion as in Figs. 5 and 6. The choroidal lesion masks fluorescence from the underlying large choroidal vessels.

Table 4 summarises the angiographic findings in patients with chorioretinitis. ICG angiography provided more detailed information about the nature of the choroidal involvement than the accompanying fluorescein angiography. Lesions that appeared to be actively inflamed on a basis of clinical history and ophthalmoscopic appearance showed hypofluorescence on ICG angiography. More detailed consideration of these hypofluorescent areas suggested two possible pathologies. In the most common form, the hypofluorescence appeared to be due to masking of the choroidal fluorescence by infiltrate, presumably inflammatory. Larger, deeper choroidal vessels could clearly be seen in transit posterior to the lesions (case 3, Figs. 5-7). Less commonly, the hypofluorescence appeared to be due to areas of choroidal hypoperfusion. The larger, deeper choroidal vessels were clearly delineated in these areas, suggesting loss of overlying choriocapillaris (case 4, Figs. 8-12). Only one chorioretinal lesion in one patient demonstrated faint fluorescence on ICG angiography in the late ( $40 \mathrm{~min}$ ) pictures and the significance of this is uncertain. ICG angiography additionally helped to determine the nature of a juxtapapillary inflammatory lesion in a patient with probable sarcoid uveitis. Fluorescein angiography findings of early and increasing fluorescence suggested an inflammatory choroidal neovascular membrane within the lesion. A corresponding hyperfluorescent area in the $30 \mathrm{~min}$ stage of ICG angiography corroborated the presence of a neovascular membrane.

Table 2. Summary of cases 1-4 (see Figs. 1-12)

\begin{tabular}{lclll}
\hline Case no. & Age (years) & Sex & Race & Diagnosis \\
\hline 1 & 41 & Female & Afro-Caribbean & Idiopathic chorioretinitis \\
2 & 32 & Male & Asian & Ischaemic retinal vasculitis \\
3 & 20 & Female & Afro-Caribbean & Idiopathic chorioretinitis \\
4 & 47 & Male & Caucasian & Idiopathic chorioretinitis \\
\hline
\end{tabular}




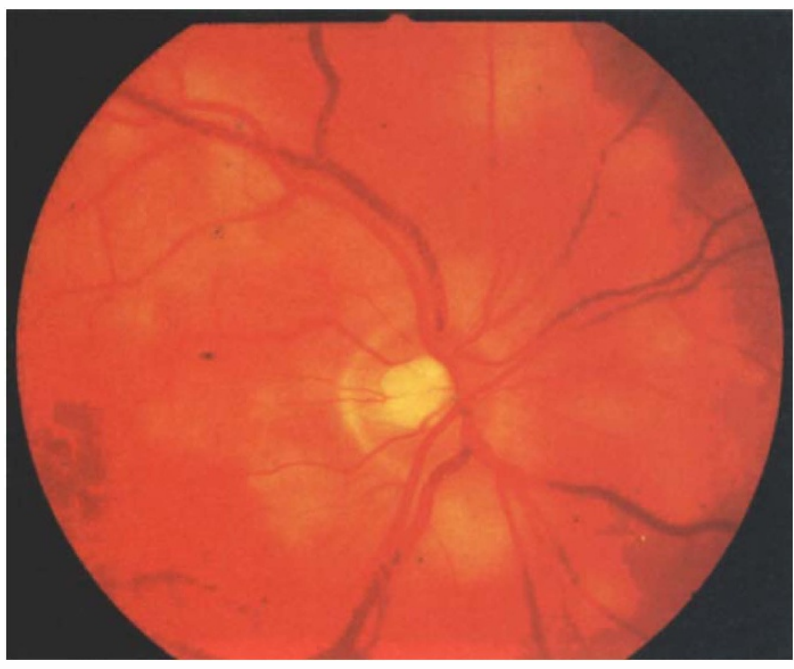

Fig. 8. Case 4: idiopathic choroiditis. Colour photograph showing the right peripapillary area. Multiple choroidal lesions are visible that appear clinically active.

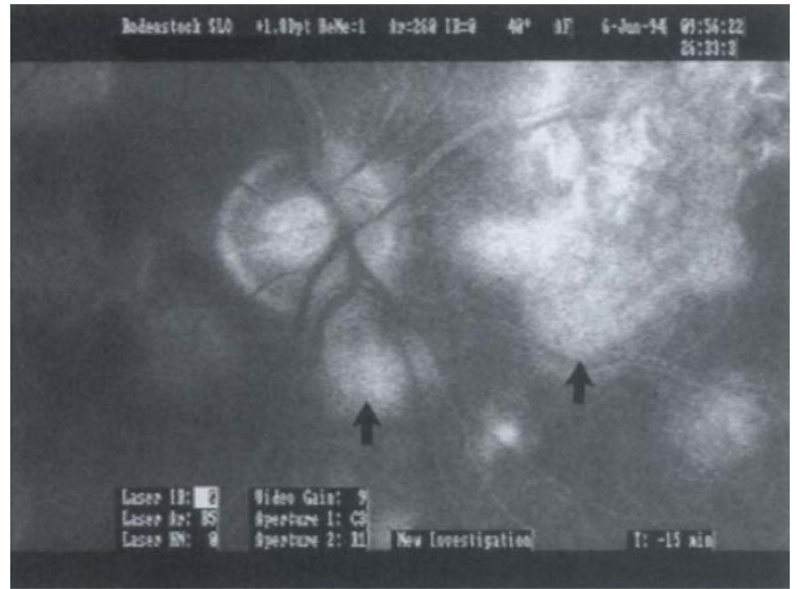

Fig. 10. Case 4: idiopathic choroiditis. Fluorescein angiogram showing late hyperfluorescence of the active lesions (arrows).

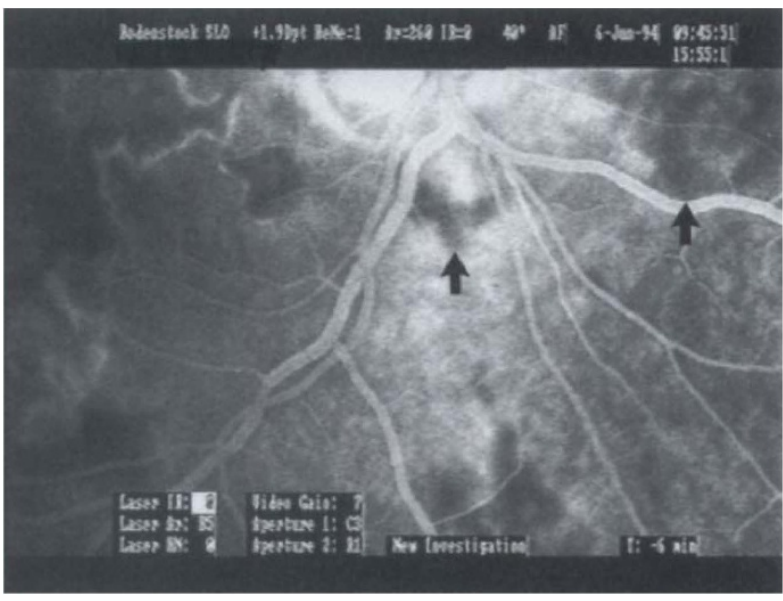

Fig. 9. Case 4: idiopathic choroiditis. Fluorescein angiogram showing early hypofluorescence of the active lesions (arrows).

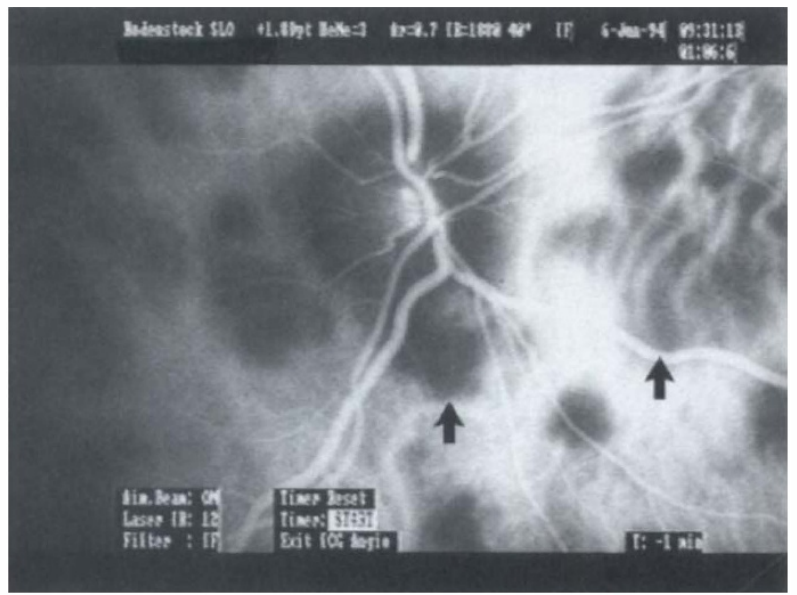

Fig. 11. Case 4: idiopathic choroiditis. ICG angiogram showing multiple dark areas that correspond to the lesions seen clinically (arrows indicate same lesions highlighted on fluorescein angiography for comparison). Large choroidal vessels are clearly seen underlying the active lesion nasal to the optic disc.

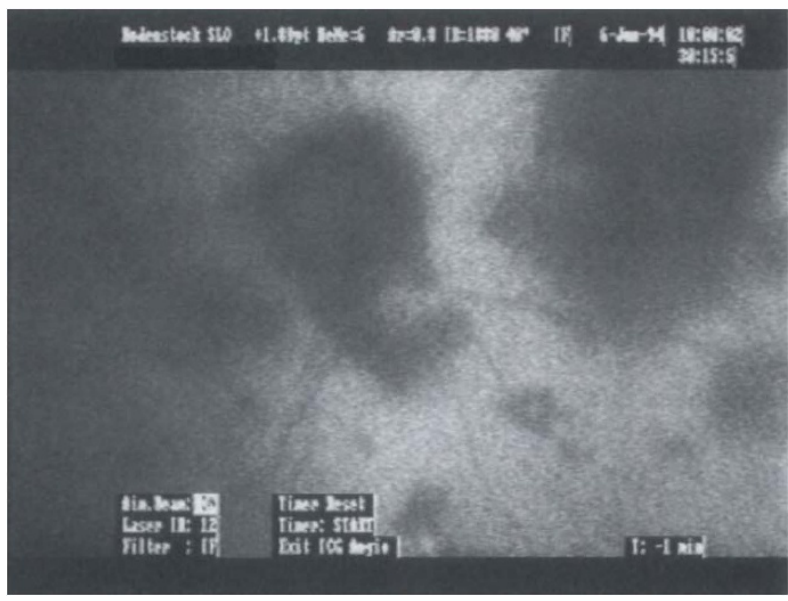

Fig. 12. Case 4: idiopathic choroiditis. Late ICG angiogram showing persistence of the hypofluorescent areas.

Old, inactive changes in the retinal pigment epithelium (RPE) and choroid of chorioretinal scarring and atrophy behaved as would be predicted on fluorescein angiography (Table 3), that is with variable window and masking defects. On ICG angiography areas of pronounced RPE hyperpigmentation masked the fluorescence from the underlying choroid. Areas of chorioretinal scarring and atrophy corresponded to welldemarcated areas of choriocapillaris loss with the underlying large choroidal vessels clearly visible. 
Table 3. Results of fluorescein and indocyanine green angiography in inflammatory eye disease with retinal signs

\begin{tabular}{lll}
\hline Retinal abnormality & Fluorescein angiographic findings (no. of patients) & ICG angiographic findings (no. of patients) \\
\hline Periphlebitis & Fluorescein leakage from retinal vein (4) & $\begin{array}{l}\text { No ICG leakage (4) } \\
\text { Staining of inflamed vascular segment (1) }\end{array}$ \\
$\begin{array}{l}\text { Retinal oedema } \\
\text { Retinal ischaemia }\end{array}$ & $\begin{array}{c}\text { Diffuse fluorescein leakage (12) } \\
\text { Hypofluorescent area due to capillary drop-out with } \\
\text { fluorescein leakage from traversing vessels (4) }\end{array}$ & $\begin{array}{c}\text { No ICG leakage and normal underlying } \\
\text { choroid (4) }\end{array}$ \\
& $\begin{array}{c}\text { flummatory disc swelling } \\
\text { Fluorescein leakage from the disc (6) }\end{array}$ & No leakage (6) \\
\hline
\end{tabular}

\section{Discussion}

Two major factors have hindered the more precise determination of the role of the choroid in inflammatory eye disease in man. Firstly, fluorescein angiography only provides limited information on the choroid in vivo. Secondly, most pathological material available for study in man necessarily comes from eyes so severely damaged by the inflammatory process that they have required enucleation and are therefore in the end stages of the disease process. ICG angiography has provided the opportunity to examine the choroid in man in vivo at different stages of disease.

ICG was not seen to leak from any ocular vessels in this study. In particular, areas that leaked fluorescein did not leak ICG (compare Fig. 1 with Fig. 2 and Fig. 10 with Fig. 11), indicating that ICG leakage is not a reliable sign of inflammatory activity. Sodium fluorescein by contrast, is a sensitive indicator of the increased blood-retinal barrier permeability that occurs during the inflammatory process and is an invaluable tool for assessing both the site and, to a certain extent, the degree of ocular inflammation. ICG is only a slightly larger molecule than sodium fluorescein but it is more highly protein bound in serum $^{12}$ (98-99\% compared with $70 \%$ respectively) so would be expected to leak from vessels much less readily. ICG is bound to a range of serum proteins, ${ }^{12-14}$ chiefly lipoprotein and albumin, and this binding effectively increases the molecular weight of ICG from $775 \mathrm{Da}$ to $66-350 \mathrm{kDa}$. Studies have been performed aimed at quantifying the vascular permeability changes that occur in uveitis using fluorescein-labelled dextrans of differing molecular weights. Dextrans of up to $70 \mathrm{kDa}$ leaked from inflamed retinal vessels and of up to $150 \mathrm{kDa}$ from the optic disc. ${ }^{15,16}$ Given these observations and the fact that frank leakage of ICG has been demonstrated from inflamed retinal vessels in a rat model of uveitis ${ }^{17}$ it is perhaps surprising that in this study ICG leakage was not seen to occur. Frank ICG leakage from retinal vessels or the optic disc has not been reported even in areas of manifest fluorescein leakage. ${ }^{18,19}$ Ill-defined late hyperfluorescence on ICG angiography, presumably due to choroidal leakage of ICG, has been reported as an inconsistent finding in clinically active areas in serpiginous choroiditis ${ }^{20}$ and birdshot choroidopathy, ${ }^{18,19}$ while late pooling of the dye has been reported, again inconsistently, in

Voyt-Koyanagi-Harada's disease (VKH). It therefore appears that, in contrast to sodium fluorescein, leakage of ICG is an unreliable indicator of areas of breakdown in the blood-ocular barriers that occur in inflammation.

ICG angiography did clearly demonstrate areas of inflammation of the choroid in this study and demonstrated these in greater detail than fluorescein angiography. The most common findings on fluorescein angiography that correspond to areas of active choroiditis are of early hypofluorescence with late hyperfluorescence - a combination of angiographic signs considered characteristic of active choroidal inflammation, ${ }^{21,22}$ and illustrated in this study by Figs. 9 and 10. Active areas of choroidal inflammation were seen on ICG angiography as areas of hypofluorescence (Figs. 7, 11 and 12; Table 3). Dark areas on ICG

Table 4. Results of fluorescein and indocyanine green angiography in inflammatory eye disease with retinal pigment epithelial and choroidal signs Choroidal abnormality Fluorescein angiographic findings (no. of patients) ICG angiographic findings (no. of patients)

Active choroiditis

Inflammatory choroidal neovascular membrane

Chorioretinal scarring and atrophy with RPE irregularity (a) Lesions hypofluorescent early and hyperfluorescent by late phase (5)

(b) Lesions not demonstrated early but hyperfluorescent by late phase (3)

(c) Miscellaneous fluorescein angiography findings of early hypofluorescence only (1), early and increasing fluorescence (1) and hypofluorescent areas with different areas becoming fluorescent late on (1)

Early phase and increasing fluorescein leakage (1)

Window and masking defects (11) (a) Masking of choroidal fluorescence by inflammatory infiltrate (4), with combination of infiltrate and choriocapillaris loss (1)

(b) Dark areas due to mixture of choriocapillaris loss and masking of choroidal fluorescence by inflammatory infiltrate (3)

(c) Masking of choroidal fluorescence by inflammatory infiltrate (3)

Late hyperfluorescence due to membrane staining with ICG (1)

Well-demarcated dark areas due to choriocapillaris loss with large underlying choroidal vessels visible (12) 
angiography corresponding to clinically active choroiditis have now been consistently reported in a wide range of posterior inflammations and choroidopathies including toxoplasmosis retinochoroiditis, ${ }^{23}$ serpiginous choroidopathy, ${ }^{20}$ birdshot choroidopathy ${ }_{1}^{19}$ multiple evanescent white dot syndrome (MEWDS), ${ }^{24,25} \mathrm{VKH}^{26-28}$ and acute posterior multifocal placoid pigment epitheliopathy (APMMPE). ${ }^{29-31}$

The question then arises as to what pathology these dark areas represent. Dark areas on ICG angiography could firstly arise from areas of reduced dye influx, i.e. reduced choroidal perfusion. Secondly dark areas could arise when dye influx is normal but pathological changes in the choroid and/or RPE result in masking of normal choroidal fluorescence. $^{25,30}$ The hypofluorescent areas on ICG angiography in this study were most commonly due to masking, with obvious masking of the fluorescence from large underlying choroidal vessels (Figs. 7 and 11). This could be ascribed to granuloma formation within the choroid as has been shown histopathologically in a number of inflammatory diseases. ${ }^{22}$ Similar ICG angiographic findings have been reported in a case of presumed choroidal tuberculoma. ${ }^{32}$ The suggestion that an area of hypofluorescence could be created by a choroidal infiltrate is further supported by an identical ICG angiographic appearance being produced by a variety of choroidal tumours. ${ }^{33}$ Choriocapillaris loss in areas of active choroiditis was suggested on ICG angiography in a few patients in this study: within the corresponding hypofluorescent area the large underlying choroidal vessels were seen in increased contrast when compared with those in surrounding normal areas. ICG angiography has previously demonstrated localised choriocapillaris loss within the placoid lesions of $\mathrm{APMPPE}^{25}$ while transient, more generalised choroidal hypoperfusion has been demonstrated in both APMPPE $^{29}$ and $\mathrm{VKH}^{26}$ It has been postulated in both these disorders that the perfusion deficits may be secondary to a choroidal vasculitis. Examination of the dark areas seen on ICG angiography provides more detailed information on the structure of fundal white areas seen clinically in a wide range of posterior inflammations and choroidopathies. The ICG angiographic findings are not incompatible with the unifying hypothesis of Ben Ezra and Forrester, ${ }^{22}$ which proposes that choroidal white dots are 'the clinical expression of microgranuloma'. These findings do suggest, however, that in some white dot syndromes choroidal ischaemic changes may predominate.

The choroidal vessels in all the patients in this study with primarily retinal disease appeared normal on ICG angiography, indicating that there were no macroscopic changes in the choroidal angioarchitecture in this subgroup of patients. Clearly, however, this does not preclude choroidal involvement in the pathogenesis of retinal vasculitis where many submicroscopic changes, such as alteration in the expression of cell adhesion molecules of the choriocapillaris, ${ }^{7}$ have been demonstrated.
This study has investigated the role of ICG angiography in a range of inflammatory eye diseases in man. ICG angiography appears to be a useful adjunct to fluorescein angiography in further elucidating in vivo the pathogenetic mechanisms in intraocular inflammation.

L.H. was supported by a Research Fellowship from the Research (Endowment) Fund of St Thomas' Hospital, London, UK. This work was further supported by the Iris Fund for the Prevention of Blindness, London, UK. The authors wish to thank Mr Hong Woon, FRCOphth, for performing some of the angiography and Mr David Spalton, FRCS, FRCOphth and Mr Michael Sanders, FRCS, FRCOphth for inclusion of a number of their patients in this study.

\section{References}

1. Causes of blindness and partial sight among adults in England and Wales 1990-1991. Office of Population Censuses and Surveys. London: HMSO, 1995.

2. Suttorp-Schulten MSA, Rothova A. The possible impact of uveitis in blindness: a literature survey. $\mathrm{Br} \mathrm{J}$ Ophthalmol 1996;80:844-8.

3. Forrester JV. Duke Elder lecture. New concepts in the role of autoimmunity in the pathogenesis of uveitis. Eye 1992;6:433-46.

4. Nussenblatt RB. Experimental autoimmune uveitis: mechanisms of disease and clinical therapeutic indications. Invest Ophthalmol Vis Sci 1991;32:3131-41.

5. Dick AD. Experimental approaches to specific immunotherapies in autoimmune disease: future treatment of endogenous posterior uveitis? Br J Ophthalmol 1995;79:81-8.

6. Dua HS, McKinnin A, McMenamin PG, Forrester JV. Ultrastructural pathology of the 'barrier sites' in experimental autoimmune uveitis and experimental autoimmune pinealitis. Br J Ophthalmol 1991;75:391-7.

7. Whitcup SM, Chi-Chao C, Qian L, Nussenblatt RB. Expression of cell adhesion molecules in posterior uveitis. Arch Ophthalmol 1992;110:662-6.

8. Muller-Hermelink HK, Kraus-Mackiw E, Daus W. Early stage of human sympathetic ophthalmia. Arch Ophthalmol 1984;102:1353-7.

9. Forrester JV, Liversidge J, Dua HS, Towler H, McMenamin PG. Comparison of clinical and experimental uveitis. Curr Eye Res 1990;S9:75-84.

10. Bischoff PM, Flower RW. Ten years' experience with choroidal angiography using indocyanine green dye: a new routine examination or an epilogue? Doc Ophthalmol 1985;60:235-91.

11. Scheider A, Schroedel C. High resolution indocyanine green angiography with a scanning laser ophthalmoscope [letter]. Am J Ophthalmol 1989;108:458-9.

12. Fox IJ, Wood EH. Indocyanine green: physical and physiological properties. Staff Meetings Mayo Clin 1960;35:732-44.

13. Baker KJ. Binding of sulfobromophthalein (BSP) sodium and indocyanine green (ICG) by plasma $\alpha_{1}$ lipoproteins. Proc Soc Exp Biol 1966;122:957-63.

14. Nakagawa $S$, Nagata $K$, Nagashima $H$. Investigation of the hepatic excretion mechanism of indocyanine green in patients with liver disease. III. Binding of indocyanine green to plasma proteins, with particular reference to the clinical evaluation. Kumamoto Med J 1977;30:9-15.

15. Atkinson EG, Jones S, Ellis BA, Dumonde DC, Graham E Molecular size of retinal vascular leakage determined by FITC-dextran angiography in patients with posterior uveitis. Eye 1991;5:440-6. 
16. Lightman SL, Caspers-Velu LE, Hirose S, Nussenblatt RB, Palestine AG. Angiography with fluorescein-labeled dextrans in a primate model of uveitis. Arch Ophthalmol 1987;105:844-8.

17. Howe LJ, Stanford MR, Dewhirst RR, Whiston R, Marshall J. Angiographic abnormalities of experimental uveoretinitis. Curr Eye Res 1996;15:1149-55.

18. Herbort CP, Borruat F-X, de Courten C, Jaccard L. Angiographie au vert d'indocyanine dans les uveites posterieures. Klin Monatsbl Augenheilkd 1996;208:321-6.

19. Howe LJ, Stanford MR, Graham EM, Marshall J. Choroidal abnormalities in birdshot chorioretinopathy: an indocyanine green angiography study. Eye 1997;11:554-9.

20. Giovanni A, Mariotti C, Ripa E, Scassellati-Sforzolini B. Indocyanine green angiographic findings in serpiginous choroidopathy. Br J Ophthalmol 1996;80:536-40.

21. Sharp DC, Bell AR, Patterson E, Pinkerton RMH. Sympathetic ophthalmia: histopathologic and fluorescein angiographic correlation. Arch Ophthalmol 1984;102:232-5.

22. Ben Ezra D, Forrester JV. Fundal white dots: the spectrum of a similar pathological process. Br J Ophthalmol 1995;79:856-60.

23. Auer C, Bernasconi O, Herbort CP. Toxoplasmic retinochoroiditis: new insights provided by indocyanine green angiography. Am J Ophthalmol 1997;123:131-3.

24. Ie D, Glaser BM, Murphy RP, et al. Indocyanine green angiography in multiple evanescent white-dot syndrome. Am J Ophthalmol 1994;117:7-12.
25. Obana A, Kusumi M, Miki T. Indocyanine green angiographic aspects of multiple evanescent white dot syndrome. Retina 1996;16:97-104.

26. Yuzawa M, Kawamura A, Matsui M. Indocyanine green video-angiographic findings in Harada's disease. Jpn J Ophthalmol 1993;37:456-66.

27. Matsunaga H, Takashi M, Fukushima I, Uyama M. Indocyanine green fluorescence angiography in Harada disease. Nippon Ganka Gakkai Zasshi 1994;98:852-7.

28. Oshima Y, Harino S, Hara Y, Tano Y. Indocyanine green angiographic findings in Vogt-Koyanagi-Harada disease. Am J Ophthalmol 1996;122:58-66.

29. Dhaliwali RS, Maguire AM, Flower RW, Arribas NP. Acute posterior multifocal placoid pigment epitheliopathy: an indocyanine green angiographic study. Retina 1993;13:317-25.

30. Howe LJ, Woon H, Graham EM, Fitzke F, Bhandari A, Marshall J. Choroidal hypoperfusion in acute posterior multifocal placoid pigment epitheliopathy: an indocyanine green angiographic study. Ophthalmology 1995;102:790-8.

31. Howe LJ. Indocyanine green angiography of acute posterior multifocal placoid pigment epitheliopathy [letter]. Ophthalmology 1996;103:863.

32. Berinstein DM, Gentile RC, McCormick SA, Walsh JB. Primary choroidal tuberculoma. Arch Ophthalmol 1997;115:430-1.

33. Shields CL, Shields JL, De Potter P. Patterns of indocyanine green videoangiography of choroidal tumours. $\mathrm{Br} \mathrm{J}$ Ophthalmol 1995;79:237-45. 